\title{
Document Image Retrieval Based on Texture Features and Similarity Fusion
}

\author{
Fahimeh Alaei \\ School of ICT, Griffith \\ University, Australia \\ fahimeh.alaei@griffithuni.edu.au
}

\author{
Alireza Alaei \\ School of ICT, Griffith \\ University, Australia \\ alireza20alaei@yahoo.com
}

\author{
Michael Blumenstein \\ University of Technology \\ Sydney, Australia \\ Michael.Blumenstein@uts.edu.au
}

\author{
Umapada Pal \\ CVPR Unit, Indian \\ Statistical Institute, India \\ umapada@isical.ac.in
}

\begin{abstract}
In this paper we investigate the usefulness of two different texture features along with classification fusion for document image retrieval. A local binary texture method, as a statistical approach, and a wavelet analysis technique, as a transform-based approach, are used for feature extraction and two feature vectors are obtained for every document image. The similarity distances between each of the two feature vectors extracted for a given query and the feature vectors extracted from the document images in the training step are computed separately. In order to use the properties of both features, a classifier fusion technique is then employed using a weighted average fusion of distance measures obtained in relation to each feature vector. The document images are finally ranked based on the greatest visual similarity to the query obtained from the fusion similarity measures. The Media Team Document Database, which provides a great variety of page layouts and contents, is considered for evaluating the proposed method. The results obtained from the experiments demonstrate a correct document retrieval of $65.4 \%$ and $91.8 \%$ in the Top-1 and Top-10 ranked document list, respectively.
\end{abstract}

Keywords: Document image retrieval; Texture features; Local binary pattern; Wavelet transform; Classifier fusion

\section{INTRODUCTION}

Document image retrieval (DIR) is an approach to detect identical or similar document images for a given query in a database [5]. The impetus is to have a paperless world, so processing a large number of document images requires efficient document image retrieval methods using discriminative features. The retrieval process using appearance-based features of a document image instead of the content of the document image is called a recognition-free approach. These approaches usually do not recognize the textual part of a document; in contrast, the retrieval process is based on the similarity of documents [3]. Different similaritybased DIR methods have been developed in the literature. The DIR methods have been grouped into different categories including layout structural similarity using visual features [1, 2]; page layout similarity using graph and XY-tree representation [3, 4]; signature-based methods and shape coding [5]; object recognition [6]; and density distributions using bag-of-visual-words features [7]. These methods usually have some notable difficulties, as the documents should have structured layouts or uniformity.

Texture is one of the important features that can show intensities in an image or in the selected region of an image. Moreover, important information about the structural arrangement and their relationship to the adjacent area can be provided using texture features [8]. Texture features represent the images in a simple but a unique form; therefore, they can be effectively employed for classification and retrieval problems. Thus, many researchers from the document image analysis (DIA) community have used various texture features in different applications, such as segmentation, layout analysis, and recognition of different document analysis systems [9-11]. The texture analysis approaches are usually grouped into different categories such as: a) statistical approaches, which consider the relationship between the greylevels of an image and do not try to understand the hierarchical concept of the texture; b) structural approaches, which consider the hierarchy of spatial arrangements of the texture besides the well-defined textural elements; c) modelbased approaches, which try to interpret an image texture using fractal and stochastic models, as well as an empirical model of each pixel generated in the image; and d) transformbased approaches which are related to the characteristics of a texture and can convert the image into a new form and domain $[13,14]$.

To retrieve pictures in historical documents texture approaches have been applied in [9]. From different parts of a page at different resolutions, five features related to frequencies and orientations have been considered for retrieval purposes. In [10], characterization of the content of a document image has been carried out without considering the structure and characteristics of treated pictures. Measurement of the similarity of documents to a query image based on query-by-example has been computed. Texture features such as visibility and complexity have been provided to determine the visual similarity of document images. For the segmentation and classification of document images texture approaches have also been considered in [12, 17]. Text and graphic content have been characterized in two different textures in the documents. A better classification performance has been provided using supervised segmentation when supervised and un-supervised segmentation schemes have been considered [17]. In [16], the discrete wavelet transform has been applied due to the multi-resolution analysis properties of the method. By applying the spectral-based retrieval model an overall document score based on magnitude and phase, which were used for occurrence and position of the terms in the pattern, have been obtained. In [18], the wavelet transforms and texture features were used for off-line signature verification. A grey level co-occurrence matrix (GLCM) has been applied to the details coefficients matrices, which were generated using the wavelet transform.

To the best of our knowledge, little attention has been paid to texture features for document image retrieval. In this paper the efficiency of two different texture features along with classifier fusion for document image retrieval is investigated. This study attempts to present a DIR method using texture 
features, which are conceptually simple and easy to implement. Texture in text regions and non-text regions are generally different in the document image, therefore texture analysis can likely be an efficient feature extraction technique for document image retrieval [12]. For these reasons, two methods from different groups of texture analysis, which are efficient texture features due to their high performance in the literature, are considered [15, 16]. First, the local binary pattern method, which is a statistical approach and summarizes local grey-level structure, is considered. Second, wavelet transform, which is a transform-based approach and generates under-sampled images, is employed. As a result, for each document image two feature vectors are obtained. The similarity distances between each of the two feature vectors extracted for a given query and the feature vectors extracted from the document images in the training step are computed separately. In order to use the properties of both features, a classifier fusion technique using the average fusion is then applied. The document images are finally ranked based on the greatest visual similarity to the query obtained from the fusion similarity measures. The Media Team Database is considered for evaluating the proposed method.

The rest of this paper is organized as follows. In Section II, the proposed approach for document image retrieval is explained. Section III is devoted to experimental analysis based on the texture feature methods, which are applied to document image retrieval. Finally, conclusions and future work are presented in Section IV.

\section{PROPOSED APPROACH}

The steps involved for document image retrieval in this research work are demonstrated in Figure 1. The proposed approach includes two phases, training and testing. In the training phase, initially, the texture features are extracted from the whole document image by applying the local binary pattern method and the wavelet transform separately, without considering any pre-processing or segmentation methods. The features which are extracted using two different methods from the training document images are then kept as knowledge based information. In the testing phase, for a given query image, the same feature extraction techniques used in the training phase are employed to characterize the query image. Similarity distances between the features, extracted from the query image and the knowledge-based information obtained during the training phase are computed for each feature. Next, in order to combine impact of both feature extraction techniques, classifier fusion with the average function by giving different weight to each similarity measure is considered. Finally, the relevant image(s), which have maximum visual similarity to the query image, are retrieved accordingly.

\section{A. Feature Extraction}

Texture approaches have been used in computer vision and pattern recognition problems in the literature including face recognition and remote sensing [19-21]. In this research work texture feature, particularly, the local binary patterns (LBP) and the wavelet transform are considered as feature extraction methods.

\section{1) Local Binary Pattern}

The local binary pattern texture feature [15, 23] has received a great deal of attention for various computer vision problems and has shown its efficiency in different applications [19-22]. The LBP method has powerful discrimination, low computational complexity, and less sensitivity to changes in illumination [24]. In [23], the LBP method has been introduced as a means of summarizing local grey-level structure. In each image (I), the patch on a circle with a radius of $(\mathrm{R})$ and difference between the grey values of a centre pixel (C) and the grey values of its neighbourhood $(\mathrm{N})$, have been considered.

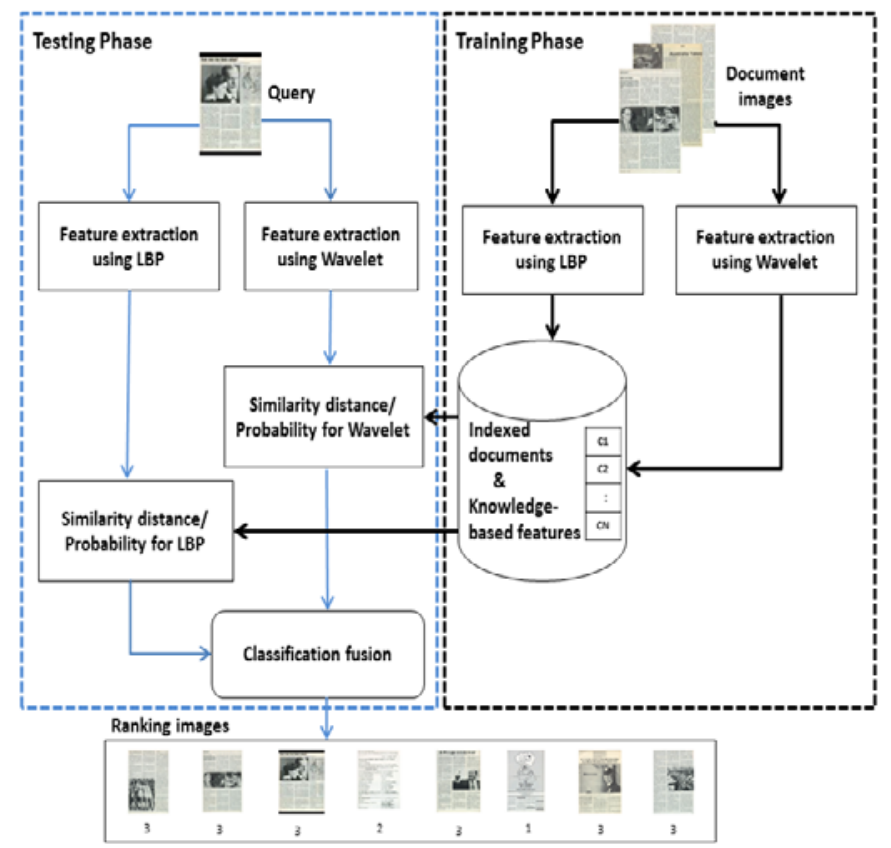

Figure 1. Block diagram of the proposed method

Different patch sizes can produce circularly symmetric neighbour sets for different values of $(\mathrm{N}, \mathrm{R})$. The values of $\mathrm{N}$ $=\{4,8,12,16,24\}$ and $\mathrm{R}=\{1,1,1.5,2,3\}$, respectively, have been taken into account for defining the patches. By considering the number of neighbours, the $\mathrm{LBP}_{\mathrm{N}, \mathrm{R}}$ method produces $2^{\mathrm{N}}$ output values which correspond to the different binary patterns. It produces a binary pattern based on the surrounding pixels. When the number of transactions between 0 and 1 of the sequence is less than or equal to two, a binary pattern is called uniform, which is an important concept in the LBP. However, there is a similarity between LBP operators and $\mathrm{LBP}_{8,1}$, since they form a circular chain [23].

In each patch the central pixel value has been considered as a threshold for that particular patch. Furthermore, the result of the binary valued image patch, as a local image descriptor, is placed in the central pixel. Thus, the LBP operator result has been made a rotation invariant, performing $n-1$ bitwise shift operations on the binary pattern and can be depicted as:

$$
\begin{aligned}
L B P_{N, R} & =\sum_{n=0}^{n-1} 2^{n} s\left(i_{n}-i_{c}\right) \\
s\left(i_{n}-i_{c}\right) & = \begin{cases}1 & \text { if }\left(i_{n}-i_{c}\right) \geq 0, \\
0 & \text { otherwise }\end{cases}
\end{aligned}
$$

Here $n$ includes the neighbours of the central pixel $c$, $i_{c}$ and $i_{n}$ are the grey-level values at $c$ and $n$. Let $S$ represent a matrix of $3 * 3$ whereby neighbour pixels are indexed.

$$
\mathrm{S}=\left[\begin{array}{lll}
i_{7} & i_{6} & i_{5} \\
i_{0} & i_{c} & i_{4} \\
i_{1} & i_{2} & i_{3}
\end{array}\right]
$$

The LBP results which are shown in Table I are based on $\mathrm{LBP}_{8,1}$. In the previous investigation, better results are obtained from $\mathrm{LBP}_{8,1}$ compared to other varieties of LBP methods. Thus, in this paper only the $\mathrm{LBP}_{8,1}$ method is considered. 
2)

Wavelet Transform

The wavelet transform method is a progressive method of signal and image analysis which can decompose the signal with finite energy in the spatial domain. The wavelet transform has been applied already for image analysis, remote sensing, noise removal [25-27], and the text retrieval method [16]. The hypothesis is that, by generating under-sampled images or in other words, applying a multi-resolution theory [28] using the wavelet transforms, the retrieval results can be augmented. The reason is, some features which are unobserved in one resolution can be observed or detected in other resolution. In this regard, the image pyramids, sub-band coding and the Haar transform are the imaging-related operators, all associated with multi-resolution analysis. Thus, the Haar transform [28] which is the simplest known orthonormal wavelet is selected for this research work [29].

Wavelet transforms are based on small waves called wavelets. A wavelet is defined by a function $\psi(x) \in L^{2}(R)$ where $L^{2}(R)$ satisfy $\int|f(x)|^{2} d x<\infty$, and has limited length and varying frequencies.

Feature extraction using the wavelet transform requires the calculation of the coefficient distribution over the mother wavelet $[18,30]$. The mother wavelet $\psi(x)$, translated by $u$ and scaled by $s$ can be represented as:

$$
\psi_{u, s}(x)=\frac{1}{\sqrt{s}} \psi\left(\frac{x-u}{s}\right)
$$

The continuous wavelet transform $W_{\psi}(u, s)$, is not helpful in practical application and our interest is to extract a new set of features based on the discreet wavelet transform (DWT) coefficients.

$$
\begin{gathered}
W_{\psi}(u, s)=\int_{-\infty}^{+\infty} f(x) \psi_{u, s}(x) d x \\
u=(k-l), s=(k-1) 2, k=1,2,
\end{gathered}
$$

These wavelets are identified by indices $l$ and $k$, and denoted by $\psi_{l k}$. To compute the wavelet coefficients of a discrete signal all integrals are replaced by sums. The $s$ and $u$ parameters are used to discretise the wavelet transform. For the argument of the scaled and transformed wavelet, equation (4) remains integer, likewise $u$ remains an integer multiple of $s$. An arbitrary signal $f(x)$ of finite energy using an orthonormal wavelet can be written as:

$$
f(x)=\sum_{k} \sum_{l} d_{l}^{k} \psi_{l}^{k}(x)
$$

where an expansion coefficient has been given by:

$$
d_{l}^{k}=\int_{-\infty}^{\infty} f(x) \psi_{l}^{k}(x) d x
$$

By applying a separate filter bank to the image the twodimensional discrete wavelet transform is computed [30]:

$$
\begin{aligned}
L_{n}\left(u_{i}, u_{j}\right) & =\left[H_{x} *\left[H_{y} * L_{n-1}\right]_{\downarrow 2,1}\right]_{\downarrow 1,2}\left(u_{i}, u_{j}\right) \\
D_{n 1}\left(u_{i}, u_{j}\right) & =\left[H_{x} *\left[G_{y} * L_{n-1}\right]_{\downarrow 2,1}\right]_{\downarrow 1,2}\left(u_{i}, u_{j}\right) \\
D_{n 2}\left(u_{i}, u_{j}\right) & =\left[G_{x} *\left[H_{y} * L_{n-1}\right]_{\downarrow 2,1}\right]_{\downarrow 1,2}\left(u_{i}, u_{j}\right) \\
D_{n 3}\left(u_{i}, u_{j}\right) & =\left[G_{x} *\left[G_{y} * L_{n-1}\right]_{\downarrow 2,1}\right]_{\downarrow 1,2}\left(u_{i}, u_{j}\right)
\end{aligned}
$$

where $H$ and $G$ are a low pass and high pass filters, and '* denotes the convolution operator; $\downarrow 2,1 \quad(\downarrow 1,2)$ denotes subsampling along the rows (columns); and $L_{n}$ is referred to as the low resolution image at scale $n$.
The equations (8-11) generate an approximation coefficients matrix which comprises a coarse summary of the original image and three details coefficients matrices, in which during decomposition, some data are lost. Subsequently, a hierarchy of these images can be generated when this process is applied iteratively to the approximation images. Using three detail images, grey-level variation or functional variation intensity of the images in different direction is measured. The directional sensitivity is a significant feature of the detail images $D_{n i}$ (horizontal, vertical, and diagonal) which are generated by filtering in a specific direction at scale $n$. Therefore, these under-sampled images are more relevant in order to characterize the features from document images. Figure 2 shows an example of the approximation coefficients and details coefficients matrices which are obtained from a sample document image.
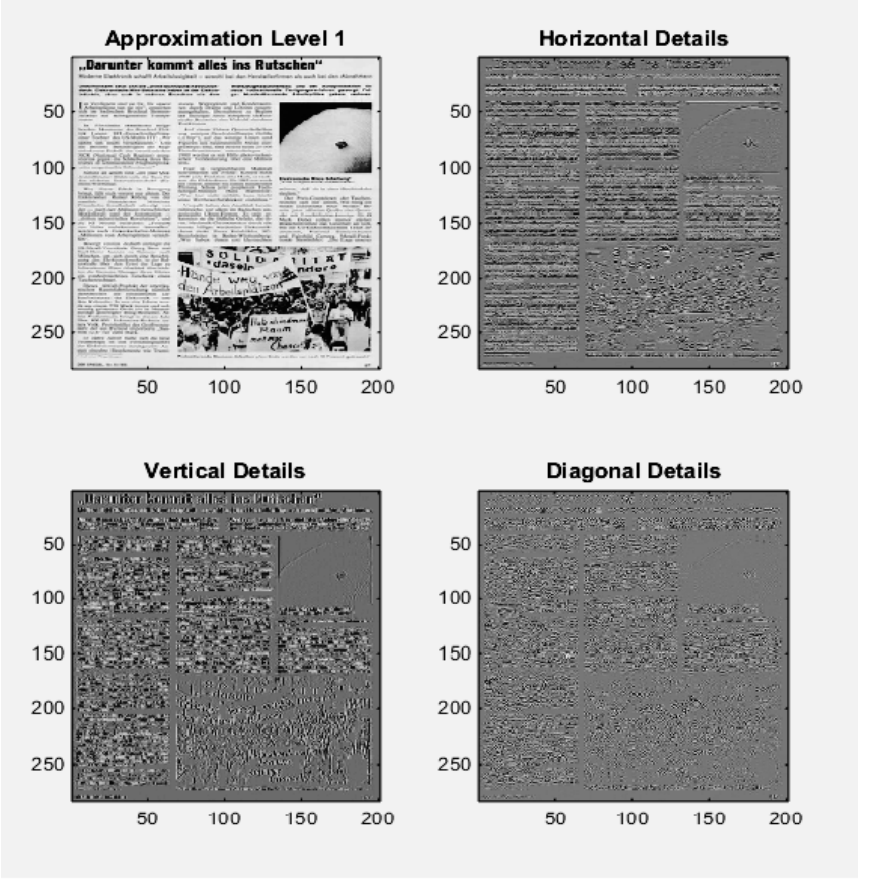

Figure 2. Example of wavelet transformation of a sample

\section{B. Creation of Knowledge-based Feature}

The global features and a global representation such as a histogram of a document image are provided using the LBP method. The feature histogram contains 59 bins when $L B P_{8,1}$ is applied for characterizing the document image. As a result, a feature vector of size 59 is extracted based on the LBP feature extraction method.

The wavelet transform that can generate multi-resolution images is employed at this level. From each resized image $(140 * 90)$, three details coefficients matrices (DCM) of size $(71 * 45)$ with wavelet decomposition are created. Then, variances of each matrix are extracted column-wise and concatenated. For more efficiency the energy from each DCM also added to the feature vector. As a result $(3 * 45)+4=139$ features are obtained. By doing so, more information from the image can be preserved in the training phase for creation of the knowledge-based features to be used for the retrieval purposes.

\section{Similarity Measure and Fusion Strategy}

To measure the similarities between a query image and the knowledge-based features obtained from the trained samples, the nearest neighbour-based methods are considered. In the 
literature nearest neighbour methods demonstrated good results for documents with complex layouts [31]. Different similarity measures have been used in the literature for computing similarities between a given query and document images in the training database. In this research work we used the Tanimoto distance based method [32] as we obtained better results compared to the Euclidean distance in both feature extraction methods. To compute the similarity measure based on the Tanimoto distance measure the following equation is used:

$$
d_{T}(A, B)=\frac{\sum_{1}^{p}(A . B)}{\sum_{1}^{p}\left|A^{2}\right|+\sum_{1}^{p}\left|B^{2}\right|+\sum_{1}^{p}(A . B)}
$$

where $d_{T}(A, B)$ is the distance between feature vectors $A$ and $B$, and $A$ and $B$ are the feature vectors extracted from a query and a document image from the database, respectively, and $p$ is the number of features in the feature vector.

When a query is given to the system, the distance of a query image and all the trained documents are computed individually according to each feature extraction method, using the Tanimoto distance. Since various feature sets are generated using different applied methods and the intention is not to depend on a single decision, classifier fusion is considered for making the decisions by combining the individual opinions. So, in order to have the properties of both methods for better decisions, classifier fusion [33, 34] is applied in this step. In other words, classifier fusion is used to obtain more efficient and accurate results. Combining classifiers basically can occur in two situations. In the first scenario, different classifiers used the same feature set for classification. In the second scenario, different feature sets can be employed using a specific classifier for classification to analyse the behaviour of different features for classification using a classifier. The emphasis of the proposed method is to consider the second situation which feature sets provided by LBP and wavelet transform separately and nearest neighbour with Tanimoto distance is employed for finding the minimum distance. For considering the posterior probability such as

$$
d^{\prime}{ }_{a}(y)=F\left(d_{a, b}(y), \ldots . d_{a, b}(y)\right), b=1,2
$$

where $b$ is the number of classifiers, $a$ varies from 1 to the number of samples used for training and $F$ stands for the minimum, maximum, average, median, or majority vote as the fusion function. Since the average function performed more accurate retrieval results among the other functions, the average function is chosen for the proposed method. If the output of classifier $d_{b}$ for a sample $a$ is $p_{a, b}=d_{a, b}(y)$ the average fusion method provide $P_{\mathrm{a}}=\frac{1}{b} \sum_{\mathrm{m}=1}^{b} p_{\mathrm{a}, \mathrm{m}}$. The distances which are obtained using LBP and the wavelet transform features for a given query image and the training sample $a$ are hereafter, respectively, called $p_{\mathrm{a}, \mathrm{LBP}}$ and $p_{\mathrm{a}, \mathrm{WT}}$. In this research work, we proposed to use a weighted average function for combining the classifiers as follows.

$$
\begin{gathered}
P_{\mathrm{a}}=\alpha * p_{\mathrm{a}, \mathrm{LBP}}+\beta * p_{\mathrm{a}, \mathrm{WT}} \\
\beta=1-\alpha
\end{gathered}
$$

where $\alpha$ and $\beta$ are the corresponding weights for the distances computed based on the classifiers. These weights are decided by experiment and fixed as $\alpha=\frac{1}{3}$ and $\beta=\frac{2}{3}$. Though, changing the value of weights could provide small variations in the results. The computed new distances based on weighted average function are further sorted in the descending order to rank the distances. The most similar image with highest similarity distance is then selected to represent the most similar image in the training database to the given query.

\section{EXPERIMENTAL ANALYSIS}

\section{A. Database and Evaluation Metric}

The Media Team Document Database (MTDB), which is comprised of heterogeneous document images, was considered to evaluate the proposed technique [35]. Numerous types of scanned documents such as address lists, advertisements, articles, business cards etc. are available in the database. There is a great diversity of page layout and contents in the database, in addition to unbalanced data in each class. Each document has a high-resolution, lowresolution and thumbnail size resolution image available in the database, whereby all the resolutions are considered for testing purpose. In our experiments, we focused on eleven of nineteen predefined classes from the MTDB that contain mainly text documents. The other 8 classes were not considered, as only a few samples (2 documents in the class) are available in the database and the samples are graphics type (music notes and maps).

Different experiments considering different training and testing sets were performed. The training and testing sets do not have any overlapping in the experiments. Only one sample from each group of document images was selected for training the proposed retrieval method. As a result, in each experiment only 154 images were considered for training and the remaining 1168 images were taken into account for testing. We chose different resolutions of the images for the training of the system in each experiment. The results obtained based on different features and the proposed fusion strategies are demonstrated in Tables I, II, and III.

The evaluation of the system is performed based on the results obtained from the proposed method considering the first (Top-1) Top-3, Top-5, and Top-10 similar documents to a query document image.

\section{B. Results and Discussion}

The MTDB includes three resolutions of document images. Therefore, the system is trained separately by three different resolution images each time to see the effect of image size and resolution on texture features for retrieval purposes. Considering that in all the experiments, only $11.6 \%$ of the data is taken for training. Among the LBP methods with different $(\mathrm{N}, \mathrm{R})$ which are applied for feature extraction, we noted that the $\mathrm{LBP}_{8,1}$ method provides better results. Thus,

\begin{tabular}{|c|c|c|c|c|}
\hline $\begin{array}{ll}\text { Training sets } & \text { Samples }\end{array}$ & $\begin{array}{c}\text { Top-1 } \\
(\%)\end{array}$ & $\begin{array}{c}\text { Top-3 } \\
(\%)\end{array}$ & $\begin{array}{c}\text { Top-5 } \\
(\%)\end{array}$ & $\begin{array}{c}\text { Top-10 } \\
(\%)\end{array}$ \\
\hline High-resolution & 57.2 & 64.7 & 67.9 & 69.4 \\
\hline Low-resolution & 36.2 & 48.4 & 66.9 & 71.5 \\
\hline Thumbnail size & 38.1 & 46.4 & 47.2 & 49.2 \\
\hline
\end{tabular}
only the results obtained from $\mathrm{LBP}_{8,1}$ method are listed in Table I.

TABLE I. THE RESULTS OBTAINED USING THE LBP ${ }_{8,1}$ METHOD.

In Table I, it can be noted that by considering highresolution document images for training, the $\mathrm{LBP}_{8,1}$ method provides a correct document retrieval of $57.2 \%$ when only the top one similar document images to the queries are considered for evaluation. Retrieval accuracy increases to $69.4 \%$ in the Top-10 similar document images to the query image.

The results obtained from the wavelet transform features are shown in Table II. It is interesting to note that lowresolution document images for training provide a $62.1 \%$ 
accuracy, which is a better result compared to high-resolution images that provide $61.5 \%$ retrieval accuracy in the first top similar document to the query images.

One of the advantages of the wavelet transform is the ability to generate multi-resolution images, which can be helpful for retrieval in this research work. So, this technique provides better results compared with the LBP method which only considers grey-levels of the pixels for the retrieval procedure.

TABLE II. THE RESULTS OBTAINED USING THE WAVELET TRANSFORM METHOD

\begin{tabular}{|c|c|c|c|c|}
\hline $\begin{array}{ll}\text { Training sets } & \text { Samples }\end{array}$ & $\begin{array}{c}\text { Top-1 } \\
(\%)\end{array}$ & $\begin{array}{c}\text { Top-3 } \\
(\%)\end{array}$ & $\begin{array}{c}\text { Top-5 } \\
(\%)\end{array}$ & $\begin{array}{c}\text { Top-10 } \\
(\%)\end{array}$ \\
\hline High-resolution & 61.5 & 77.5 & 82.9 & 88.1 \\
\hline Low-resolution & 62.1 & 77.8 & 83.2 & 88.0 \\
\hline Thumbnail size & 39.9 & 67.9 & 76.4 & 89.5 \\
\hline
\end{tabular}

Applying classifier fusion and giving weight to the classifiers, in order to have a more accurate retrieval system can be noted as a contribution of this work. In one part, the LBP method as a statistical texture feature provides global features and a global representation of a document image by means of a histogram. In other part, the wavelet analysis as a transform-based method, generate the multi-resolution images. The wavelet transform can be estimated and reduce the global influence of grey-level variation. Considering the advantages of both the methods, the obtained results based on the proposed method has shown better retrieval accuracy. The results obtained using classifier fusions are illustrated in Table III.

TABLE III. THE RESULTS OBTAINED USING THE PROPOSED METHOD

\begin{tabular}{|c|c|c|c|c|}
\hline $\begin{array}{ll}\text { Training sets } & \text { Samples }\end{array}$ & $\begin{array}{c}\text { Top-1 } \\
\text { (\%) }\end{array}$ & $\begin{array}{c}\text { Top-3 } \\
\text { (\%) }\end{array}$ & $\begin{array}{c}\text { Top-5 } \\
(\%)\end{array}$ & $\begin{array}{c}\text { Top-10 } \\
\text { (\%) }\end{array}$ \\
\hline High-resolution & 64.0 & 78.2 & 83.9 & 89.1 \\
\hline Low-resolution & 65.4 & 78.0 & 82.9 & 88.6 \\
\hline Thumbnail size & 47.6 & 70.9 & 81.1 & 91.8 \\
\hline
\end{tabular}

By looking at Table III, it can be noted that low-resolution images used for training provide a $65.4 \%$ accuracy in the first top similar document to the query. Nonetheless, this system in the Top-10 images could achieve $91.8 \%$ accuracy, which is a promising result. Figure 3 illustrates that the retrieval results using classifier fusion provide a higher level of accuracy in the three resolutions.

The results of the $\mathrm{LBP}_{8,1}$ method were compared with the results of the proposed method using both $\mathrm{LBP}_{8,1}$ and wavelet features. It can be seen that, in the first top samples, using high-resolution, low-resolution, and thumbnail size training set, $6.8 \%, 29.2 \%$ and $9.5 \%$ improvements, respectively, were occurred when proposed method were applied. By comparing the results of wavelet transform with the results of proposed method, using high-resolution, low-resolution, and thumbnail size training set, $2.5 \%, 3.3 \%$ and $7.7 \%$ enhancement, respectively, were occurred when proposed method were applied. These progresses can be observed in Figure 3 for all the results. Since proposed method considered the advantage of both the method, the results are improved compared to each one of the method. Regarding computation time complexity, the proposed system is applicable for real scenario.

The investigation shows that when the LBP feature is applied to the DIR system using high-resolution images for training, better results are achieved compared to thumbnail size images. The reason is that, in high-resolution images enough information can be extracted to characterize the image and hence better retrieval results are obtained. Another interesting point of these experiments is the sudden increment of the results in low-resolution images when wavelet transform is applied to the system. It can be clarified that the DWT is a highly efficient decomposition method for signals and images. This property is taken into account to have multiresolution images, which can be generated from an image. Accordingly, texture features, which are undetected in one resolution, could be detected from other resolution of the images. Since different resolutions of a document are available in the MTDB for testing the proposed system, DWT could provide richer feature information to be used for document retrieval.
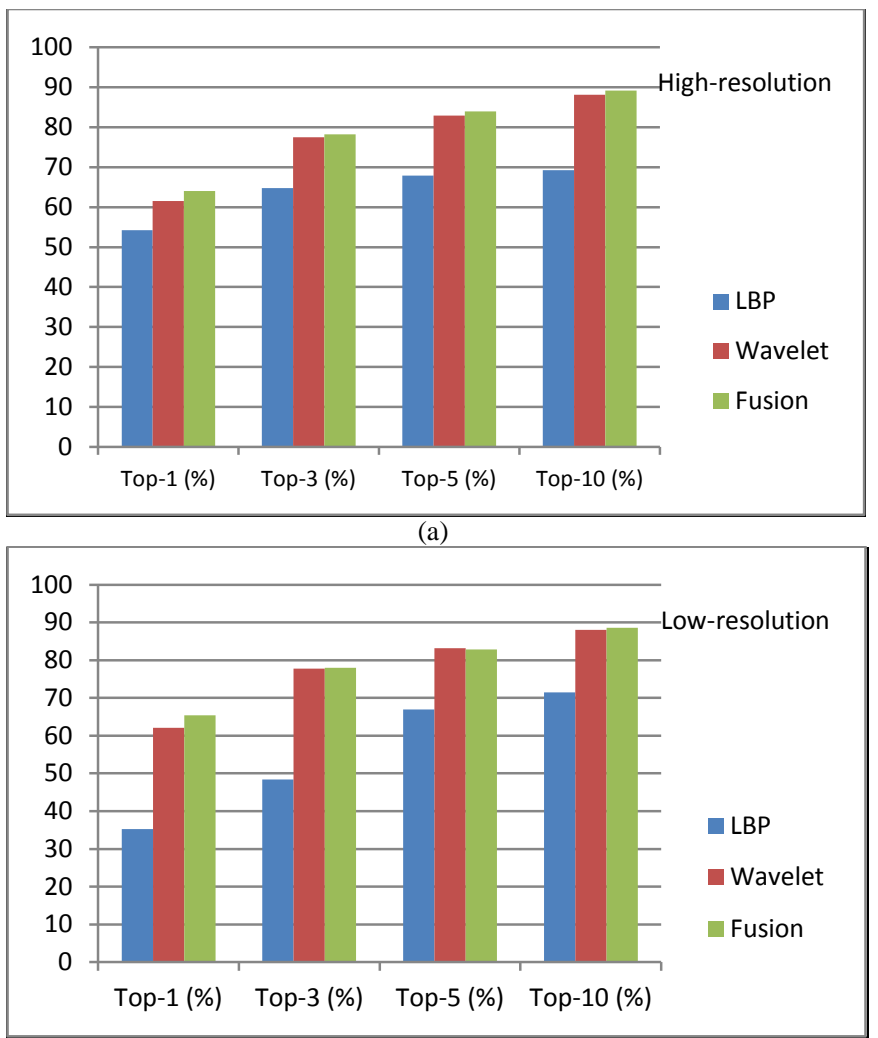

(b)

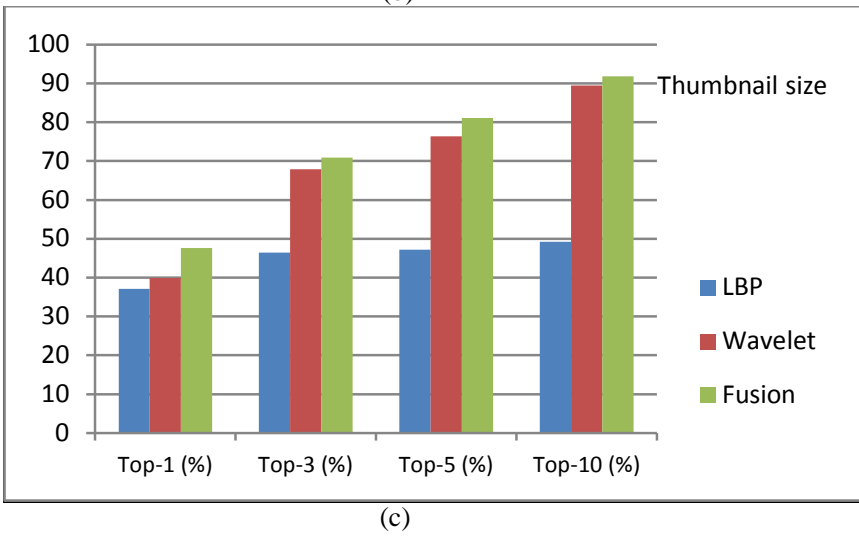

Figure 3. The results obtained from the proposed system based on different features using (a) High-resolution, (b) Low-resolution, and (c) Thumbnail size images for training.

\section{Comparative Analysis}

In [11], the MTDB has also been used for classification of documents based on transform-based methods. The wavelet transform based features and SVM classifier with the ratio of $1: 1$ for training and testing, in the case of text-only images, could provide $25.4 \%$ correct classification accuracy. For hybrid images an accuracy of $45.3 \%$ has also been recorded. The accuracy of $65.4 \%$ was obtained using our proposed method. This may not be an overall equivalent comparison of 
the results with our proposed method, since the whole database is divided into three types for evaluation. However, one of the benefits of the proposed method in this research work relates to the encouraging results attained on heterogeneous and complex databases, such as the MTDB. Moreover, more attention has been paid to structured documents for the retrieval procedure in the literature of DIR [1, 2, 6, 36, 37], whereas in this research work heterogeneous document images were used for document retrieval.

\section{CONCLUSIONS AND FUTURE WORK}

A document image retrieval method based on two different categories of texture features is studied in this paper. Firstly, the spatial arrangements of the grey-levels of the pixels in a patch of an image are described and the patch moves over the image to extract the texture features for measuring the similarity. The variances of multi-resolution images which are generated using the Haar wavelet transform are then described. It can be noted that, considering undersampled images is helpful for improving the retrieval accuracy when different resolutions of images are available in the database. The properties of both the methods are taken into account using classifier fusion. The results are improved by considering classifier fusion for document image retrieval. Furthermore, more research is required for finding the efficient set of texture features for document image retrieval, which can consider unstructured and complex documents.

\section{REFERENCES}

[1] J. Kumar, P. Ye, and D. Doermann, "Structural similarity for document image classification and retrieval”, Pattern Recognition Letters, 43: pp. 119-126, 2014.

[2] J.V. Beusekom, D. Keysers, F Shafait, and T.M. Breuel, "Distance measures for layout-based document image retrieval”, in Proceedings of the International Conference on Document Image Analysis for Libraries, pp. 242-253, 2006

[3] A. Gordo, J. Gibert, E. Valveny, and M. Rusiñol, “A kernel-based approach to document retrieval”, in Proceedings of the International Workshop on Document Analysis Systems, pp. 377-384, 2010.

[4] F. Cesarini, S. Marinai, and G. Soda, "Retrieval by layout similarity of documents represented with MXY trees", in Proceedings of the International Workshop on Document Analysis Systems, pp.353-364, 2002.

[5] J. Li, Z.G Fan, Y.Wu, N. Le, "Document image retrieval with local feature sequences", in Proceedings of the International Conference on Document Analysis and Recognition, pp. 346-350, 2009.

[6] C.L.Tan, W. Huang, Z.Yu, and Y. Xu, "Imaged document text retrieval without OCR”, Pattern Analysis and Machine Intelligence, 24(6): pp. 838-844, 2002.

[7] K. Kise, Y. Wuotang, and K. Matsumoto, "Document image retrieval based on 2D density distributions of terms with pseudo relevance feedback”, in Proceedings of the International Conference on Document Analysis and Recognition, pp. 488-492, 2003.

[8] R.M. Haralick, K. Shanmugam, and I.H. Dinstein, "Textural features for image classification”, Systems, Man and Cybernetics, 6: pp. 610-621, 1973.

[9] N. Journet, J.Y. Ramel, R. Mullot, and V. Eglin, “A proposition of retrieval tools for historical document images libraries”, in Proceedings of the International Conference on Document Analysis and Recognition, pp. 1053-1057, 2007.

[10] V. EGLIN, and S. BRES, "Document page similarity based on layout visual saliency: Application to query by example and document classification", in Proceedings of the International Conference on Document Analysis and Recognition, pp. 1208 - 1212, 2003.

[11]C.F. Tsai, “On classifying digital accounting documents”, International Journal of Digital Accounting Research, 7(13): pp. 53-71, 2007.

[12]M.W. Lin, J.R. Tapamo, and B. Ndovie, "A texture-based method for document segmentation and classification”, South African Computer Journal, 36: pp. 49-56, 2006.

[13] M.H. Bharati, J.J. Liu, and J.F. MacGregor, "Image texture analysis: methods and comparisons", Chemometrics and Intelligent Laboratory Systems, 72(1): pp. 57-71, 2004.

[14]A. Materka, and M. Strzelecki, "Texture analysis methods-a review", Technical university of lodz, institute of electronics, COST B11 Report, Brussels, pp. 9-11, 1998.

[15]T. Ojala, M. Pietikäinen, and D. Harwood, “A comparative study of texture measures with classification based on featured distributions", Pattern Recognition, 29(1): pp. 51-59, 1996.
[16]L.A. Park, K. Ramamohanarao, and M. Palaniswami, “A novel document retrieval method using the discrete wavelet transform”, ACM Transactions on Information Systems , 23(3): pp. 267-298, 2005.

[17] T Randen, and J.H. Husøy, "Segmentation of text/image documents using texture approaches”, in Proceedings of NOBIM, pp. 60-67, 1994.

[18] J.F. Vargas, C. M. Travieso, J. B. Alonso, and M.A. Ferrer, "Off-line signature verification based on gray level information using wavelet transform and texture features", in Proceedings of the International Conference on Frontiers in Handwriting Recognition, pp. 587-592, 2010.

[19]X. Tan, and B. Triggs, "Enhanced local texture feature sets for face recognition under difficult lighting conditions”, Image Processing, 19(6): pp. 1635-1650, 2010.

[20] M. Pietikäinen, T. Nurmela, T. Mäenpää, and M. Turtinen, "View-based recognition of real-world textures. Pattern Recognition”, 37(2): pp. 313323, 2004.

[21]M. Heikkilä, and M. Pietikäinen, “A texture-based method for modeling the background and detecting moving objects”, Pattern Analysis and Machine Intelligence, 28(4): pp. 657-662, 2006.

[22]A. Fernández, M.X. Álvarez, and F. Bianconi, “Texture description through histograms of equivalent patterns", Journal of Mathematical Imaging and Vision, 45(1): pp. 76-102, 2013.

[23]T. Ojala, M. Pietikäinen, and T. Mäenpää, "Multiresolution gray-scale and rotation invariant texture classification with local binary patterns", Pattern Analysis and Machine Intelligence, 24(7): pp. 971-987, 2002.

[24]M. Heikkilä,, M. Pietikäinen, and C. Schmid, "Description of interest regions with local binary patterns”, Pattern Recognition, 42(3): pp. 425436, 2009.

[25]C. Zhu, and X. Yang, "Study of remote sensing image texture analysis and classification using wavelet”, International Journal of Remote Sensing, 19(16): pp. 3197-3203, 1998.

[26]Z. Xizhi, "The application of wavelet transform in digital image processing”, in MultiMedia and Information Technology, pp. 326-329, 2008.

[27] J.P. Antoine, P. Carrette, R. Murenzi, and B. Piette,’Image analysis with two-dimensional continuous wavelet transform", Signal Processing, 31(3): pp. 241-272, 1993.

[28] A. Haar, "On the theory of orthogonal function systems", Math. Ann, 69(3): pp. 331-371, 1910.

[29]R.C. Gonzalez, and R.E. Woods, Book on "Digital image processing". Prentice-Hall of India Pvt. Ltd, 2005.

[30] S.G. Mallat, "A theory for multiresolution signal decomposition: the wavelet representation", Pattern Analysis and Machine Intelligence, 11(7): pp. 674-693, 1989.

[31] K.S. Kumar, S. Kumar, and C. Jawahar, “On segmentation of documents in complex scripts”, in proceedings of the International Conference on Document Analysis and Recognition, pp. 1243-1247, 2007.

[32]D.J Rogers, and T.T. Tanimoto, “A computer program for classifying plants”, Science, 132(3434): pp. 1115-1118, 1960.

[33] L.I. Kuncheva, "A theoretical study on six classifier fusion strategies", Pattern Analysis \& Machine Intelligence, (2): p. 281-286, 2002.

[34] J. Kittler, M. Hatef, R.P. Duin, and J. Matas, “On combining classifiers", Pattern Analysis and Machine Intelligence, 20(3): pp. 226-239, 1998.

[35] J. Sauvola, and H. Kauniskangas, "Media team document database II”, A CD-ROM collection of document images, University of Oulu Finland, 1999.

[36] S. Marinai, E. Marino, and G. Soda, "Exploring digital libraries with document image retrieval”, Research and Advanced Technology for Digital Libraries, pp. 16-21, 2007.

[37] S. Marinai, B. Miotti, and G. Soda, "Digital libraries and document image retrieval techniques: A survey”, Learning Structure and Schemas from Documents, pp. 181-204, 2011. 